\title{
Bad for the heart?
}

CD20-specific antibodies, which deplete mature B cells, are currently in clinical use for the treatment of severe rheumatoid arthritis and certain cancers. B cell activation has been proposed to be protective against atherosclerosis, and therefore it is possible that the clinical use of these antibodies could increase the risk of cardiovascular disease. However, the effect of mature $\mathrm{B}$ cell depletion on atherosclerosis in immunocompetent mice is unknown. Now, Mallat and colleagues show that depletion of mature B cells in several mouse models of atherosclerosis actually reduces the development of atherosclerotic plaques.

Previous studies have shown that loss of B cells, as a result of $\mu \mathrm{MT}$ deficiency or splenectomy, accelerated the development of atherosclerosis in mice. However, loss of other cell populations, in addition to mature $B$ cells, may have contributed to this phenotype, so, the authors assessed the effect of a CD20-specific monoclonal antibody (which depleted mature B cells but relatively preserved B-1 B cells) in several mouse models of atherosclerosis. CD20-specific antibody treatment of apolipoprotein $\mathrm{E}$ (APOE)-deficient mice, which were placed on a high-calorie or chow diet, and low-density lipoprotein (LDL) receptor-deficient mice reduced the development of atherosclerotic lesions in both models.

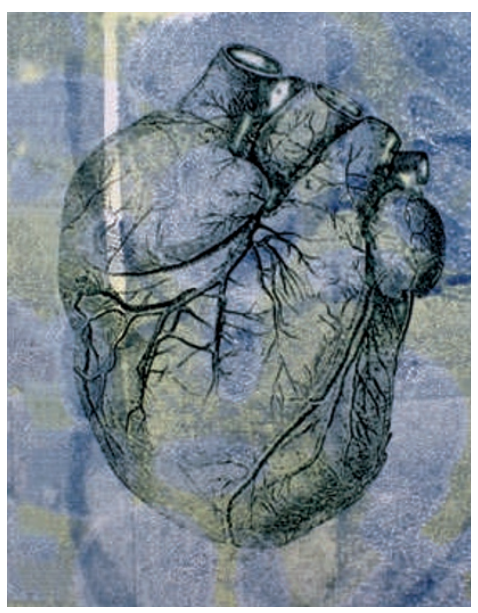

To further understand these surprising observations, the authors examined the levels of oxidized LDL (oxLDL)-specific antibodies (following CD20-specific antibody treatment) in $A p o e^{-/-}$mice that were placed on a high-calorie diet. The levels of oxLDL-specific IgG antibodies were reduced following treatment, whereas the levels of oxLDL-specific IgM antibodies, which are potentially protective, were only marginally affected. Furthermore, B cell depletion resulted in reduced activation of dendritic cells (reduced CD40 expression levels) and T cells (reduced CD44 and CD69 expression levels) in vivo and reduced the accumulation of $\mathrm{T}$ cells in atherosclerotic lesions.

Analysis of cytokine production by T cells isolated from CD20-specific antibody-treated $A p o e^{-/-}$mice revealed reduced pro-atherogenic interferon- $\gamma$ but enhanced interleukin-17A (IL-17A) production compared with control cells. Furthermore, neutralization of IL-17A reversed the protective effects of CD20-specific antibody treatment on atherosclerotic plaque development in these mice.

Therefore, the data show that $\mathrm{B}$ cells contribute to the development of atherosclerosis in mice by modulating $\mathrm{T}$ cell activation, recruitment and cytokine production.

Olive Leavy

ORIGINAL RESEARCH PAPER Ait-Oufella, $\mathrm{H}$. et al. B cell depletion reduces the development of atherosclerosis in mice. J. Exp. Med. 5 Jul 2010 (doi:10.1084/jem.20100155) 